\title{
Dickkopf-1 secreted by decidual cells promotes trophoblast cell invasion during murine placentation
}

\author{
Sha Peng ${ }^{1,2}$, Jing $\mathrm{Li}^{1,2}$, Chenglin Miao ${ }^{1,2}$, Liwei Jia ${ }^{1,2}$, Zeng $\mathrm{Hu}^{1,2}$, Ping Zhao ${ }^{1,2}$, Juxue $\mathrm{Li}^{1,2}$, \\ Ying Zhang ${ }^{1,2}$, Qi Chen ${ }^{1,2}$ and Enkui Duan ${ }^{1}$ \\ ${ }^{1}$ State Key Laboratory of Reproductive Biology, Institute of Zoology, Chinese Academy of Sciences, Datun Road, \\ Chaoyang District, Beijing 100101, China and ${ }^{2}$ Graduate School of the Chinese Academy of Sciences, Beijing \\ 100039, China \\ Correspondence should be addressed to E-K Duan; Email: duane@ioz.ac.cn
}

\begin{abstract}
Dickkopf-1 (Dkk1) is one of the secreted antagonists in the canonical Wnt signaling pathway. It plays important roles in diverse developmental processes. However, the role of Dkk1 in trophoblast cell invasion during placentation remains unclear. In this study, we found that Dkk1 was mainly expressed in maternal decidual tissue but trivially in ectoplacental cones (EPCs) in day 8 post coitum (p.c.) pregnant mouse uterus and that the efficiency of EPC attachment and outgrowth was increased when co-cultured with decidual cells, which secreted Dkk1, and this enhancement was abolished by pretreating decidual cells with Dkk1 blocking antibody before co-culture experiment. This indicates that Dkk1 secreted by decidual cells plays an important role in trophoblast cell invasion. Indeed, when recombinant mouse Dkk1 was added to EPCs in vitro, the efficiency of attachment and outgrowth was increased. Migration of EPCs toward the decidua was retarded when antisense Dkk1 oligonucleotide (ODN) was administered via intrauterine injection in vivo. Furthermore, the active $\beta$-catenin nuclear location was lost when we treated cultured EPCs with recombinant mouse Dkk1, and the efficiency of EPCs attachment and outgrowth was obviously increased when we treated cultured EPCs with antisense $\beta$-catenin ODN. Taken together, Dkk1 secreted by decidual cells may induce trophoblast cell invasion in the mouse and $\beta$-catenin may be involved in such functions of Dkk1.
\end{abstract}

Reproduction (2008) 135 367-375

\section{Introduction}

Dickkopf-1 (Dkk1), a secreted glycoprotein, inhibits canonical Wnt signaling by binding to the Wnt coreceptors LRP5 and LRP6, and to Kremen1 and Kremen2. The resulting trimolecular complex of Dkk1, LRP5/6, and Kremen is endocytosed, resulting in the depletion of LRP5/6 co-receptor from the plasma membrane (Semenov et al. 2001, Mao et al. 2002, Rothbacher \& Lemaire 2002). $\beta$-Catenin is an intracellular mediator of canonical Wnt signaling, which is phosphorylated and stabilized as a result of activation of Wnt ligand and transported to the nucleus where, as part of a transcriptional complex, it regulates gene expression (Gumbiner 1995, Huber et al. 1996, Miller \& Moon 1996, Reya \& Clevers 2005, Jarvinen et al. 2006).

During early development, Dkk1 regulates head formation in Xenopus (Glinka et al. 1998), and Dkk1 null mice do not develop head structures anterior to the midbrain (Mukhopadhyay et al. 2001). During tumorigenesis, such as colorectal cancer, mesothelioma, and medulloblastoma, Dkk1 also acts as a crucial regulator (Lee et al. 2004, Aguilera et al. 2006, Vibhakar et al.
2007). There are many similarities between tumor invasion and trophoblast cell invasion. Thus, we speculate Dkk1 may have potential functions in trophoblast cell invasion.

Mouse embryo implantation is a highly controlled event requiring invasion of the uterine stroma by the extraembryonic trophoblasts (Cross et al. 1994). On day 5.5 of gestation, cells adjacent to the inner cell mass, the polar trophectoderm, continue to proliferate and form the ectoplacental cones (EPCs). On day 6.5 of gestation, the outer cells of the EPCs begin differentiation into invasive secondary trophoblast giant cells (sTGCs). Placentation is dependent on the invasion of the uterine stroma by these cells during days 7.5-10.5 of gestation (Afonso et al. 1997). Trophoblast cell invasion is a transient process, with stringent spatial and temporal controls. Our previous study had revealed that Dkk1 and Kremen 1 receptor had positive immunostaining in EPCs and the maternal decidual compartment on day 7 in pregnant uterus (data not shown). Generally, murine trophoblast cell invasion begins from day 8 post coitum (p.c). The expression of Dkk1 on day 8 in pregnant uterus 
and its potential roles in trophoblast cell invasion during murine placentation have not been stated.

Here, we detected the Dkk1 expression in day 8 p.c. pregnant mouse uterus. A co-culture model of decidual cells and EPCs was employed in our study. We found that Dkk1 secreted by decidual cells could promote trophoblast cell invasion during murine placentation through inhibiting canonical Wnt signaling pathway. Our research could increase our knowledge of the molecular mechanisms during placentation.

\section{Results}

\section{Dkk1 was expressed and secreted from decidual cells, but not EPCs}

Our previous study had revealed that Dkk1 had positive immunostaining in the maternal decidual compartment and the EPCs on day 7 in pregnant uterus in mice (Li et al. 2007). Murine trophoblast cell invasion begins from day 8 . To explore the potential roles of Dkk1 during trophoblast cell invasion, we first examined Dkk1 expression on day 8 in pregnant uterus. As shown in Fig. 1A, Dkk1 protein expressed strongly in the maternal decidual compartment but relativity weak in EPCs. When decidual cells and EPCs were separated from uterus on day 8 of pregnancy and cultured for $24 \mathrm{~h}$, the expression of Dkk1 was maintained in decidual cells (Fig. 1E), but in EPCs, it only showed a slight staining in marginal sTGCs migrating from EPCs (Fig. 1C). The concentration of Dkk1 secreted by cultured decidual cells was $63.55 \pm 1.15 \mathrm{pg} / \mathrm{ml}$ as measured by ELISA (Fig. 1I).

\section{Dkk1 increased the efficiency of EPC attachment and outgrowth}

The process of EPC attachment and outgrowth in vitro is similar to adhesion and invasion stages of trophoblast development in utero (Sherman \& Atienza-Samols 1978, Enders et al. 1981). Therefore, we investigated the process of trophoblast cell invasion through examining EPC attachment and outgrowth in vitro.

To test the role of endogenous Dkk1 during trophoblast cell invasion, we co-cultured decidual cells and EPCs. The efficiency of EPC attachment and outgrowth was increased when co-cultured with decidual cells compared with cultured EPCs alone. But pretreatment of
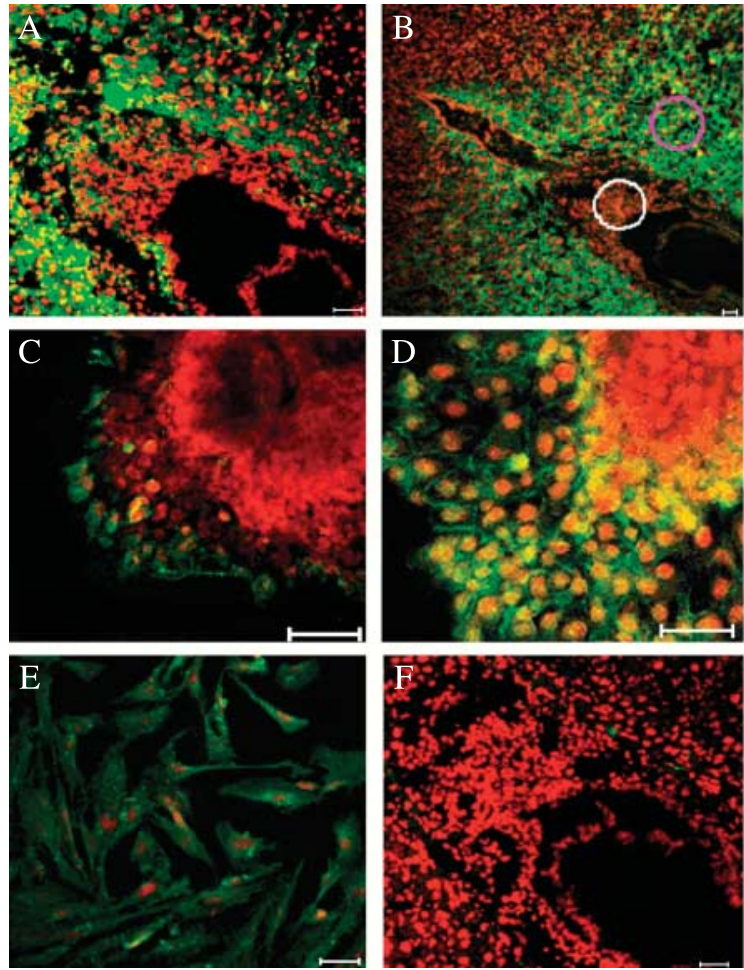

Dkk1

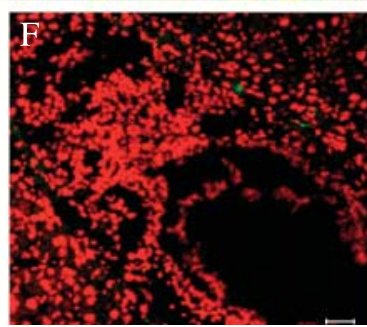

$\beta$-catenin
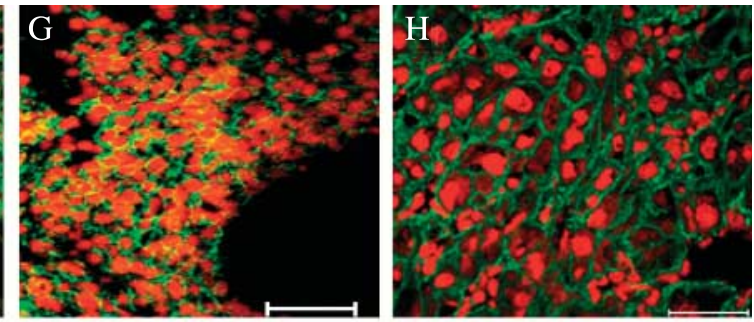

I

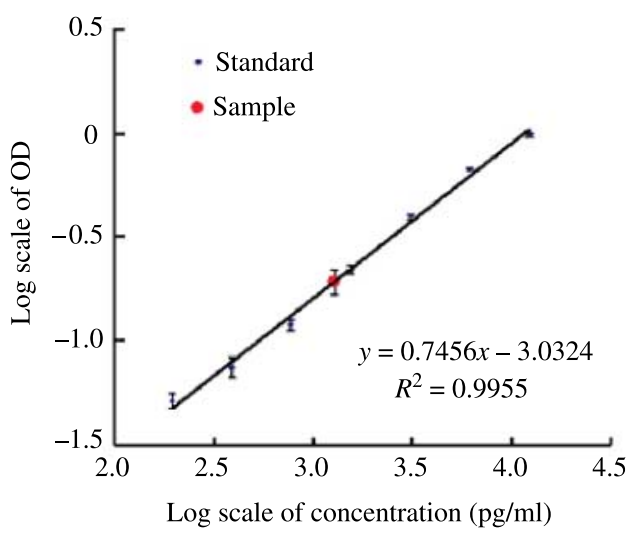

Figure 1 Immunofluorescence of Dkk1 and $\beta$-catenin on day 8 in pregnant uterus, cultured EPC, and detection of Dkk1 secreted by decidual cells through ELISA. Green signals represent Dkk1 and $\beta$-catenin staining, and red signals indicate nuclear staining. Yellow color represents an overlap of green and red signals. $B a r=50 \mu \mathrm{m}$. (A) Dkk1 expression on day 8 in pregnant uterus. $(B, G$, and $H) \beta$-Catenin expression on day 8 in pregnant uterus. (G) A higher magnification of the white circled area in (B). (H) A higher magnification of the purple circled area in (B). (C) Dkk1 expression in cultured EPC. (D) $\beta$-Catenin expression in cultured EPC. (E) Dkk1 expression in cultured decidual cells. (F) Negative control using preimmune goat IgG. (I) The standard curve was constructed by plotting the log of the Dkk1 concentrations versus the log of the OD. The score of Dkk1 was the average of results from three individual experiments. 
decidual cells with Dkk1 blocking antibody for $24 \mathrm{~h}$ decreased the efficiency of EPC attachment and outgrowth compared with the non-treatment group (Fig. 2A). This result indicates that Dkk1 secreted by decidual cells may function during trophoblast cell invasion and further supported by the fact that the role of recombinant mouse Dkk1.

To further study the roles of Dkk1 during trophoblast cell invasion, we treated EPCs in vitro with recombinant mouse Dkk1 imitating endogenous Dkk1 secreted by decidual cells. The results revealed that the efficiency of attachment and outgrowth was increased significantly after treatment with recombinant mouse Dkk1 compared with control (Fig. 2B). These results indicate that Dkk1 secreted by decidual cells may promote trophoblast cell invasion.
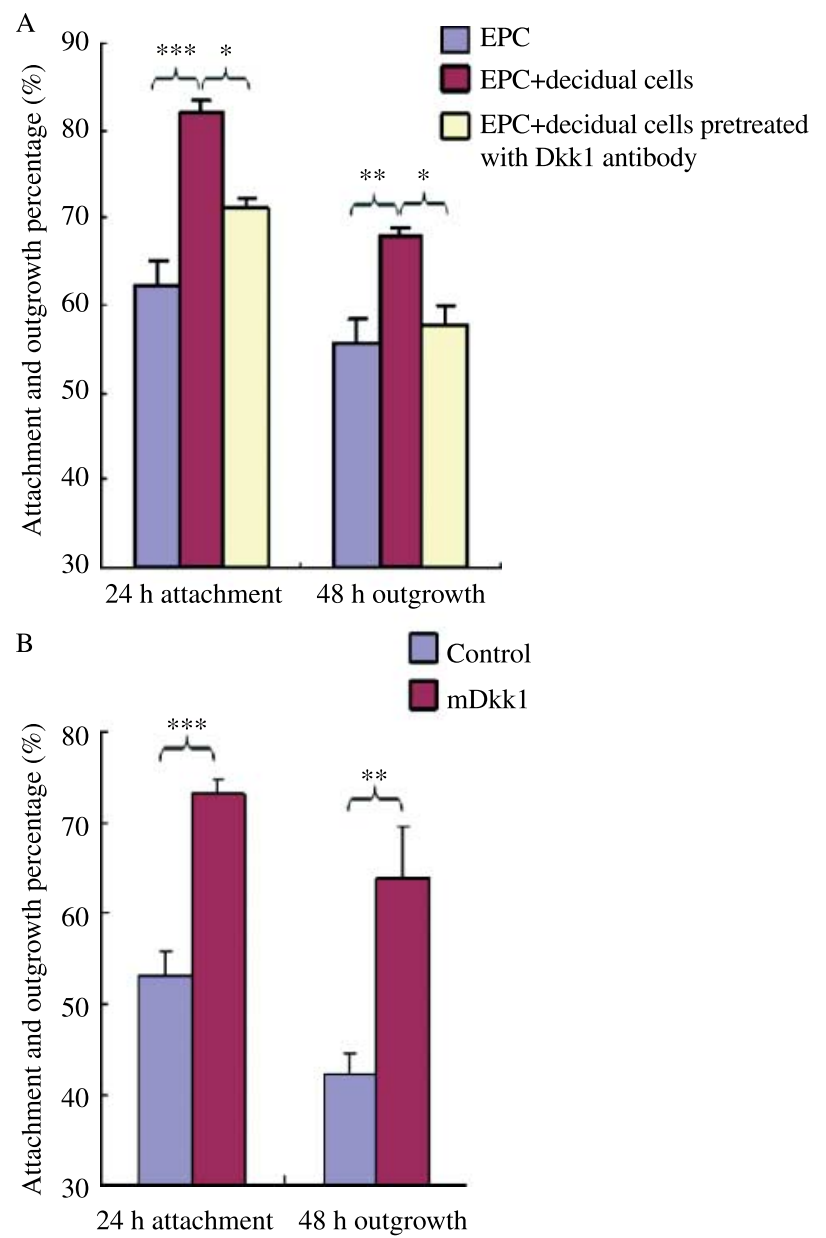

Figure 2 The effects of Dkk1 on EPC attachment and outgrowth. (A) Effect of endogenous Dkk1 secreted by decidual cells. The efficiency of EPC attachment and outgrowth after being cultured alone, co-cultured with decidual cells or pretreated decidual cells with Dkk1 blocking antibody for $24 \mathrm{~h}$ before co-culture. (B) The effect of recombinant mouse Dkk1. The efficiency of EPC attachment and outgrowth after treatment with $200 \mathrm{ng} / \mathrm{ml}$ Dkk1. Results were expressed as mean \pm S.E.M. of three replicates. ${ }^{*}, * *$ and ${ }^{* * *}$ indicate significant difference from the control $(P<0.05, P<0.01$, and $P<0.001$ respectively).

\section{Antisense Dkk1 ODN retarded the migration of sTGCs to decidual cells in vivo}

Since embryo implantation is a complex process, which happens in the evening of day 4 p.c. pregnant mouse. Trophoblast cell invasion is a key step in the formation of the placenta, taking place on day 8 p.c. In order to further study the effect of Dkk1 on trophoblast cell invasion after implantation, we used intrauterine injection on day 5 p.c. as an in vivo model (the results were detected on day 8). Before injection, we examined the efficiency of antisense Dkk1 ODN using immunostaining and RT-PCR. We found that $20 \mu \mathrm{M}$ antisense Dkk1 ODN prominently suppressed the expression of Dkk1 both at protein and RNA levels (Fig. 3A-D). As shown in Fig. $3 \mathrm{E}$ and $\mathrm{F}$, the number of implantation sites significantly decreased on the side where antisense Dkk1 ODN was injected compared with control. Hematoxylin and eosin (H\&E) staining results indicate that antisense Dkk1 ODN prevented sTGC migration toward decidual cells (Fig. 3G) compared with sense Dkk1 ODN (Fig. 3H). This suggests that Dkk1 was necessary during trophoblast cell invasion and blocking as it could suppress the sTGCs migration.

\section{Antisense $\beta$-catenin oligonucleotide (ODN) increased EPC attachment and outgrowth percentage}

We detected $\beta$-catenin expression on day 8 in pregnant uterus for exploring the molecular mechanism during trophoblast cell invasion. We found that $\beta$-catenin was located only in the membrane of decidual cells, and had slightly staining in the membrane and nuclei of sTGCs (Fig. 1B, G, and H). In cultured EPCs in vitro, $\beta$-catenin had strong staining in the nuclei of sTGCs (Fig. 1D). Generally, the canonical Wnt pathway is considered as being activated when $\beta$-catenin is expressed in the nuclei (van de Wetering et al. 2002). Therefore, we concluded that the canonical Wnt pathway was inhibited to some degree during trophoblast cell invasion.

To verify the roles of $\beta$-catenin during trophoblast cell invasion, we detected the efficiency of EPC attachment and outgrowth after treatment with antisense $\beta$-catenin ODN or sense $\beta$-catenin ODN for 24 and $48 \mathrm{~h}$. We examined the efficiency of antisense $\beta$-catenin ODN using immunostaining and RT-PCR. We found that $20 \mu \mathrm{M}$ antisense ODN prominently suppressed the expression of $\beta$-catenin both at protein and RNA levels (Fig. 4A and B). The attachment and outgrowth percentage increased significantly when we treated EPCs with $20 \mu \mathrm{M}$ antisense ODN, compared with control and sense ODN treatment (Fig. 4C). We concluded that antisense $\beta$-catenin ODN increased attachment and outgrowth percentage of EPCs, hence $\beta$-catenin possibly inhibited trophoblast cell invasion during murine placentation. 


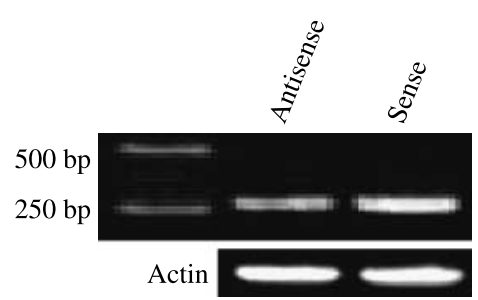

E

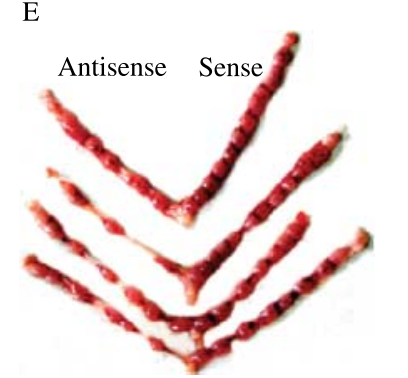

B

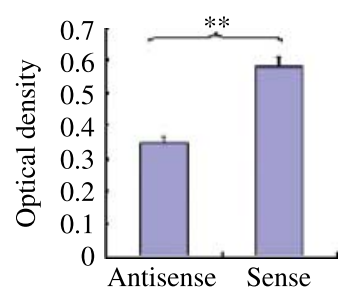

F

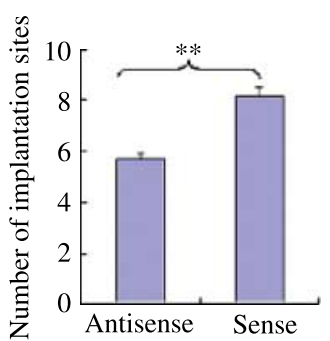

C

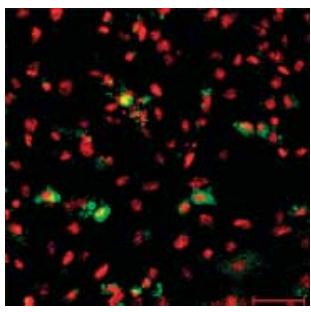

Antisense Dkk1

G

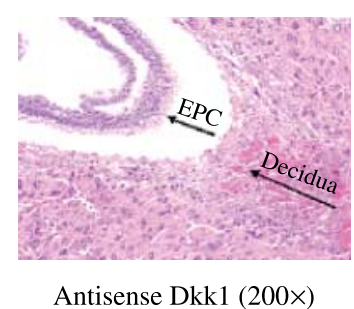

D

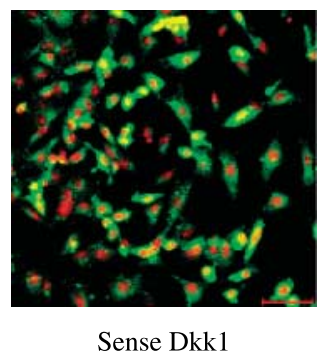

$\mathrm{H}$

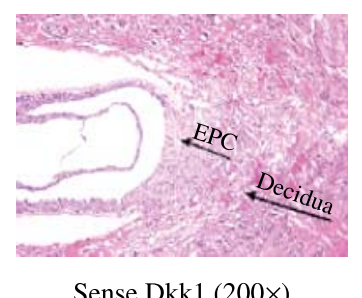

Figure 3 The effect of antisense Dkk1 ODN on the migration of sTGCs toward decidual cells in vivo. (A-D) Detection for the inhibition efficiency of antisense Dkk1 ODN at mRNA and protein levels. (A) mRNA levels of Dkk1 were detected after treatment of decidual cells with $20 \mu \mathrm{M}$ sense or antisense Dkk1 ODN. (B) Densitometric analysis of RT-PCR for Dkk1. Results are presented as mean \pm s.E.M. of three triplicates (**P<0.01). (C and D) Immunostaining of Dkk1 after treatment of mouse uterine epithelial cells with $20 \mu \mathrm{M}$ antisense (C) or sense (D) Dkk1 ODN. (E) Implantation sites on day 8 after intrauterine injection of antisense or sense Dkk1 ODN on day 5 of gestation. (F) The number of implantation sites was counted on day 8 of gestation after injection. $(G)$ and $(H) H \& E$ staining of paraffin sections of implantation sites after intrauterine injection. (G) Injection with antisense Dkk1 ODN. (H) Injection with sense Dkk1 ODN.

\section{Dkk1 inhibited nuclear location of active $\beta$-catenin in sTGCs of cultured EPCs}

To further explore the molecular mechanism during trophoblast cell invasion, we examined the localization of active $\beta$-catenin in cultured EPCs after treatment with $200 \mathrm{ng} / \mathrm{ml}$ recombinant mouse Dkk1. The result revealed the loss of nuclear location of active $\beta$ - catenin in sTGCs (Fig. 5B) compared with control (Fig. 5A). This suggested that Dkk1 augmented mouse trophoblast cell invasion, possibly through inhibition of the Wnt/ $\beta$-catenin signaling pathway.

\section{Dkk1 enhanced activity of MMP9 and inhibited proliferation of sTGCs in cultured EPCs}

Matrix metalloproteinase 9 (MMP9) is considered to be an important gelatinase that modulates the ingression of the trophoblast into the maternal decidua (Abrahamsohn \& Zorn 1993). Therefore, we investigated the effect of Dkk1 on activity of MMP9. As shown in Fig. 5C and D, when we treated EPCs with recombinant mouse Dkk1 for $24 \mathrm{~h}$, the activity of MMP9 was prominently enhanced.

Further, we detected the rate of cell proliferation after Dkk1 treatment. The results revealed that Dkk1 inhibited sTGCs proliferation significantly (Fig. 5E and F).

\section{Discussion}

The present study demonstrates that Dkk1 secreted by decidual cells promotes trophoblast cell invasion in mice. This effect is possibly through inhibiting the canonical $\mathrm{Wnt} / \beta$-catenin signaling pathway.

Dkk1 is a member of a multi-gene family that plays important functions in many developmental processes including head development in amphibian embryos (Glinka et al. 1998) and the formation of both the anterior nervous system and the axial mesendoderm in zebrafish (Hashimoto et al. 2000). It also acts as a crucial regulator in heart development of Xenopus (Foley \& Mercola 2005). But the function of Dkk1 during placentation was poorly understood. In this article, we first reported that Dkk1 could promote trophoblast cell invasion during murine placentation; this was proved through detecting EPC attachment and outgrowth percentage in vitro as well as the use of intrauterine injection in vivo.

The process of mouse embryo implantation includes trophoblast invasion in a broad sense. It involves the invasion of the embryo into uterine epithelial cells at the early stage and the invasion of the sTGCs of the EPC into the uterine decidua at the late stage (Gardner \& Papaioannou 1975, Romagnano \& Babiarz 1990). Attachment and outgrowth of both embryo and EPCs are usually used as in vitro model in studying embryo 
A

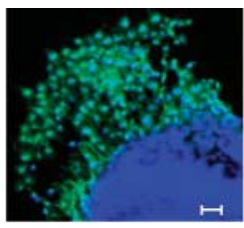

$10 \mu \mathrm{M}$

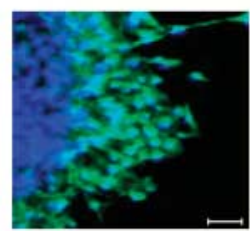

$0 \mu \mathrm{M}$

B

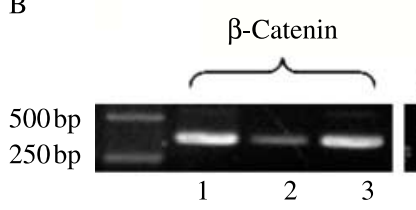

1 Control

$220 \mu \mathrm{M}$ antisense $\beta$-catenin ODN

$320 \mu \mathrm{M}$ sense $\beta$-catenin ODN

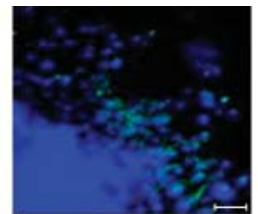

$20 \mu \mathrm{M}$

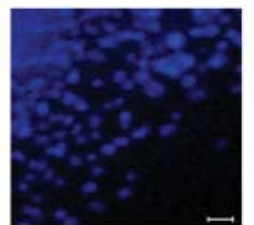

Negative control

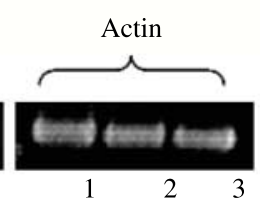

23

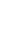

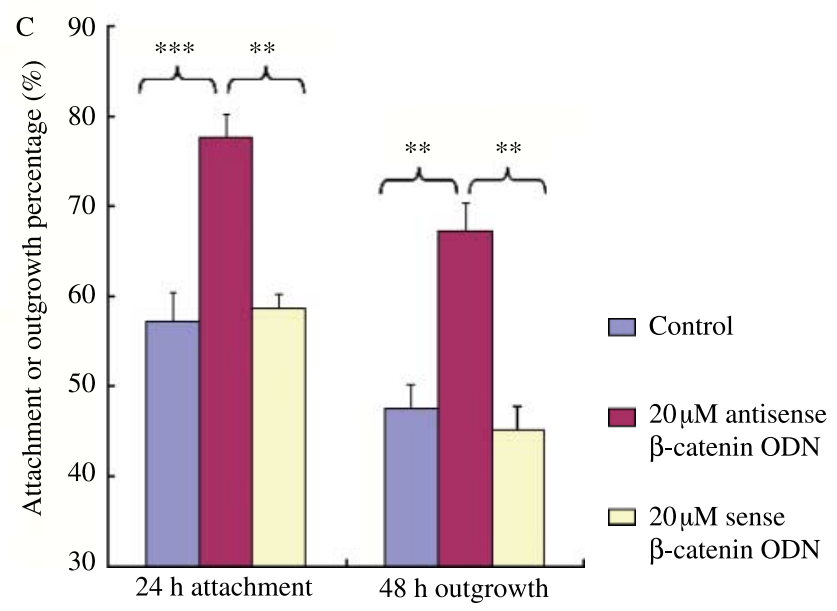

Figure 4 The effect of antisense $\beta$-catenin ODN on the efficiency of EPC attachment and outgrowth. (A and B) Detection for the inhibition efficiency of antisense $\beta$-catenin ODN at mRNA and protein levels. (A) Immunostaining of $\beta$-catenin after treatment cultured EPCs with a range of concentrations of antisense $\beta$-catenin ODN: 0,10 , and $20 \mu \mathrm{M}$. Green signals represent $\beta$-catenin staining, and blue signals indicate nuclear staining. (B) mRNA levels of $\beta$-catenin were detected without treatment or after treatment cultured EPCs with $20 \mu \mathrm{M}$ sense or antisense $\beta$-catenin ODN. (C) EPCs attachment and outgrowth percentage without treatment or after treatment with $20 \mu \mathrm{M}$ sense or antisense $\beta$-catenin ODN. Asterisks indicate significant difference $\left(* * P<0.01,{ }^{* * *} P<0.001\right)$.

implantation and trophoblast invasion (Sherman \& Atienza-Samols 1978, Enders et al. 1981). In our experiment, the efficiency of EPC attachment and outgrowth was increased when co-cultured with decidual cells compared with cultured EPCs on fibronectin (FN). It suggests that some factors secreted by decidual cells increased EPC invasion during trophoblast invasion. However, when decidual cells were pretreated with Dkk1 blocking antibody, the attachment and outgrowth of EPCs on decidual cells decreased significantly compared with the non-treatment group. Further, the recombinant mouse Dkk1 obviously increased the percentage of EPC attachment and outgrowth on FN. Therefore, Dkk1 secreted by decidual cells could increase the invasive ability of trophoblasts during placentation.

The process of trophoblast cell invasion is strictly regulated in a spatial-temporal pattern. In humans, too little or an excess of invasion can result in pregnancy abnormalities, such as preeclampsia or choriocarcinoma (Karmakar et al. 2004). In our experiment, $\beta$-catenin was slightly stained in the nuclei of sTGCs and decidual cells in day 8 p.c. pregnant mouse uterus, but had a strong staining in the nuclei of sTGCs in cultured EPC in vitro, suggesting that the $\mathrm{Wnt} / \beta$-catenin signaling pathway was inhibited in vivo but activated in vitro. However, when Dkk1 was added in the culture medium, the nuclear localization of $\beta$-catenin in EPCs was decreased significantly. The same effect was also detected when $\beta$-catenin antisense ODNs were added in the culture medium. It seems that the inhibition of $\mathrm{Wnt} / \beta$-catenin signaling pathway plays an important role in regulating trophoblast invasion. In our previous study, mutant activation of $\beta$-catenin signaling pathway significantly decreased the attachment and outgrowth of EPCs on FN. Taken together, these results indicate that inhibition of $\mathrm{Wnt} /$ signaling pathway in the embryo, especially in the trophoblast, functions importantly in both embryo implantation and placentation.

The proliferation and migration of sTGCs are tightly regulated in an autocrine/paracrine manner by numerous growth and regulatory factors within the trophoblast-endometrial microenvironment during trophoblast invasion (Bischof et al. 2001). In our experiment, Dkk1 promoted the migration but decreased the proliferation of sTGCs, which was consistent with what we observed in transformed human trophoblast cells, JEG and JAR cells (Peng et al. 2006). The process of trophoblast invasion requires the outermost layer of EPCs to differentiate into sTGCs and subsequently migrate into the uterine decidua. The arrest of cell proliferation may be the first step of trophoblast cell differentiation (Faria \& Soares 1991). In our experiment, we also found that the nuclear location of $\beta$-catenin in sTGCs is lost 


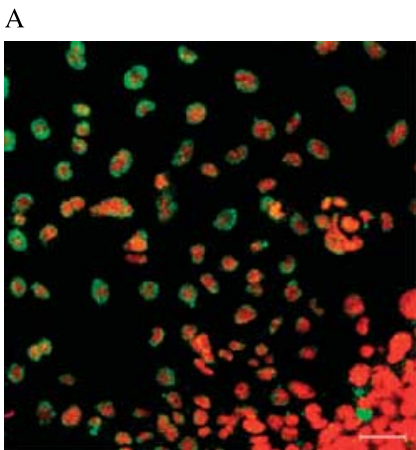

Control

C

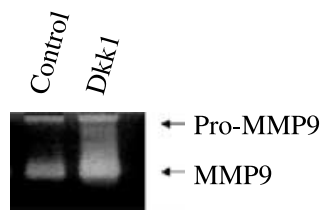

E

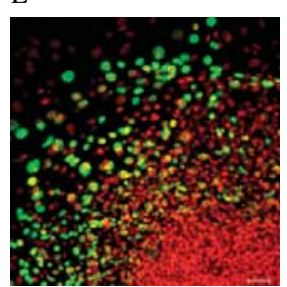

Control

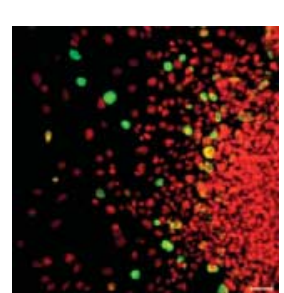

Dkk1

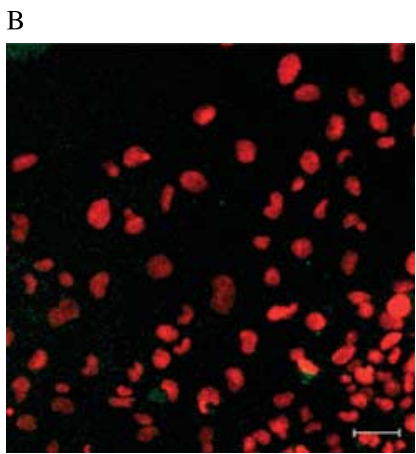

Dkk1
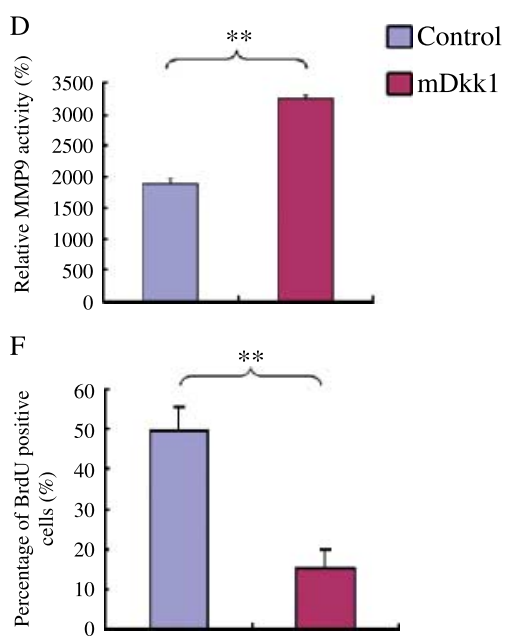

Figure 5 The effect of recombinant mouse Dkk1 on the active $\beta$-catenin expression, MMP9 activity, and proliferation of sTGCs in cultured EPCs. (A, B, and E) Green signals represent $\beta$-catenin staining, and red signals indicate nuclear staining. Yellow color represents an overlap of green and red signals. Bar $=50 \mu \mathrm{m}$. (A) Active $\beta$-catenin expression of sTGCs in cultured EPCs without treatment. (B) $\beta$-Catenin expression of sTGCs in cultured EPCs after treatment with $200 \mathrm{ng} / \mathrm{ml}$ recombinant mouse Dkk1. (C) Samples from the culture medium were analyzed for gelatinase activity. Bands correspond to relative levels of pro-MMP9 and MMP9 activities. The gel is a representation of three independent experiments. (D) Total activities of MMP9 detected by gelatin zymography were quantified by computer-aided densitometry. Results are expressed as mean \pm S.E.M. of three replicates. **Indicates significant differences from the control $(P<0.01)$. (E) The effect of recombinant mouse Dkk1 on proliferation of sTGCs in EPCs. (F) The percentage of BrdU-positive sTGCs. Results are expressed as mean \pm s.E.M. of three replicates. ${ }^{* *}$ Indicates significant differences from the control $(P<0.01)$. significantly after Dkk1 treatment. Treatment with antisense ODNs of $\beta$-catenin further verified the inhibition of the $\beta$-catenin signaling pathway associated with trophoblast invasion ability. Thus, Dkk1 secreted by maternal decidual cells increases the migration of trophoblasts by inhibiting the $\beta$-catenin signaling pathway in sTGCs.

Mutant activation of the $\mathrm{Wnt} / \beta$-catenin signaling pathway has been found in many different cancers, such as lymphoblastic leukemia, colorectal cancer, and human germ cell tumors (Fritsch et al. 2006, Herbst \& Kolligs 2007, Khan et al. 2007). Dkk1, the special antagonist of the canonical Wnt/B-catenin signaling pathway, is always referred to as a tumor suppressor gene that efficiently inhibited the proliferation of those cancer cells. A recent study demonstrated that decreased nuclear $\beta$-catenin localization and increased $\mathrm{Ki}-67$ expression may be involved in choriocarcinoma carcinogenesis (Wong et al. 2006). In our experiment, Dkk1 secreted by decidual cells increased trophoblast invasion but decreased trophoblast proliferation, indicating that the inhibition of the $\beta$-catenin signaling pathway plays a very important role in regulating trophoblast invasion. The equilibrium regulated by decidual cells, secreted Dkk1 and activity of the Wnt/ $\beta$-catenin signaling pathway in sTGCs controls trophoblast invasion during placentation. Future studies should be focused on how this equilibrium can be disturbed during some pathological conditions such as preeclampsia and choriocarcinoma.

In conclusion, our findings may shed light on a new mechanism that exists in trophoblast cell invasion during murine placentation. These data increase understanding of the mechanism of human trophoblast cell invasion and might be useful for treatment of abnormal invasion of trophoblast cells that cause disease in humans.

\section{Materials and Methods}

\section{Animals}

Kunming white strain mice were purchased from the Experimental Animal Center, Institute of Zoology, Chinese Academy of Sciences, and raised in a constant photoperiod (14 h light:10 h darkness). The Guidelines for the Care and Use of Animals in Research were followed. Mice were allowed free access to water and food. Virgin female mice were mated with fertile males of the same strain. The appearance of vaginal plug on the next day was recorded as day 1 of pregnancy. 


\section{Attachment and outgrowth assays of EPCs}

EPCs from the mice on day 8 of pregnancy were dissected out under sterile conditions and placed into FN (10 $\mu \mathrm{g} / \mathrm{ml}$; Sigma)coated four-well plates ( 30 EPCs per well). The EPCs were cultured in Ham's F-12 (Gibco) medium with 5\% fetal bovine serum (FBS) at $37{ }^{\circ} \mathrm{C}$ in an atmosphere of $5 \% \mathrm{CO}_{2}$. Recombinant mouse Dkk1 (1765-DK; R\&D Systems, Minneapolis, MN, USA), antisense or sense ODN against $\beta$-catenin (antisense $\beta$-catenin, 5'-GGAGTTTAACCACAACAGGCAGTCC- $3^{\prime}$ and sense $\beta$-catenin, $5^{\prime}$-GGACTGCCTGTTGTGGTTAAACTCC-3'; Haertel-Wiesmann et al. 2000) was supplemented to freshly isolated EPCs respectively.

In vitro attachment and outgrowth assays were performed according to the methods established by our group (Dai et al. 2003). Briefly, EPCs were incubated in FN-coated wells for 24 or $48 \mathrm{~h}$, and then shaken for $20 \mathrm{~s}$ (one rotation per second). At the end of shaking, EPCs that remained secure at the base of the wells were considered to be 'attached', whereas floating EPCs were designated as 'non-attached'. After attachment, EPCs began to grow outward. When sTGCs were visible around the attachment sites of the EPCs, we designated the EPCs as an 'outgrowth'. Outgrowth was observed using phase-contrast microscopy (Olympus, Tokyo, Japan).

The efficiencies of EPC attachment and outgrowth were evaluated as the ratios of the numbers of attached and outgrown EPCs to the number of total EPCs per well respectively. Two wells were performed per treatment. Each experiment contained at least three independent cultures.

\section{Co-culture of EPCs and decidual cells}

Decidual cells were isolated and maintained as previously described with minor modifications (Caluwaerts et al. 2000). Briefly, on day 8 of pregnancy, the mice were killed and deciduas were collected. After rinsing in PBS for three times, the deciduas were minced and digested with $1 \mathrm{mg} / \mathrm{ml}$ collagenase I (Sigma) at $25{ }^{\circ} \mathrm{C}$ for $90 \mathrm{~min}$. Digestion was stopped by the addition of two volumes of Ham's F-12 medium with $10 \%$ FBS. The dispersed cells were washed and filtered through a nylon sieve to remove the gross cone residues. The filtered cell suspension (1-2 ml) was added to Ham's F-12 medium and collected by centrifuging at $500 \mathrm{~g}$ for $10 \mathrm{~min}$, then resuspended in Ham's F-12 medium with $10 \%$ FBS. The purified decidual cells were plated on four-well plates at $1 \times 10^{6}$ cells $/ \mathrm{ml}$. After $24 \mathrm{~h}$, the decidual cells were washed thrice with PBS and followed by adding Ham's F-12 medium with $5 \%$ FBS. Then EPCs isolated as described above were seeded on decidual cells. After 24 and 48 h, EPC attachment and outgrowth were recorded respectively. Two wells were performed per treatment. Each experiment contained at least three independent cultures.

\section{Reverse-transcription PCR (RT-PCR)}

Total RNAs were isolated from decidual cells after treatment with antisense or sense Dkk1 ODN using Trizol reagent (Gibco BRL) according to manufacturer's instructions. A total of $2 \mu \mathrm{g}$ RNA was reverse transcribed by avian myeloblastosis virus reverse transcriptase (Promega) and $2 \mu \mathrm{g}$ random hexamer primers (Takara, Dalian, China). The primers used for amplifying mouse Dkk1 and $\beta$-actin were as follows. Dkk1: sense primer, 5'-GCCAGAGACACTAAACCGACAG-3' and antisense primer, 5'-GAGAAACAAGGCAATGTACCAC-3 ${ }^{\prime}$; $\beta$-actin: sense primer, $5^{\prime}$-TCTGTGTGGATTGGTGGCTCTA- $3^{\prime}$ and antisense primer, 5'-CTGCTTGCTGATCCACATCTG-3'. Amplification was performed using 30 cycles for Dkk1 and 25 cycles for $\beta$-actin. The thermal cycling conditions were as follows: $94{ }^{\circ} \mathrm{C}$ for $30 \mathrm{~s}, 53{ }^{\circ} \mathrm{C}$ for $30 \mathrm{~s}$, and $72{ }^{\circ} \mathrm{C}$ for $45 \mathrm{~s}$. Several control reactions were performed to confirm the quality of RT products and to exclude genomic DNA contamination. The experiment contained at least three independent cultures of decidual cells.

\section{Immunofluorescence staining}

Frozen sections of murine uterus, EPCs, decidual cells, and uterine epithelial cells from day 8 of pregnancy were cultured for $24 \mathrm{~h}$, fixed in $4 \%$ paraformaldehyde solution, and immunofluorescence stained. Washing thrice in PBS, the samples were permeabilized with $0.2 \%$ Triton X-100 (Sigma) for 20 min at room temperature, then rinsed several times in PBS, followed by incubation in 5\% BSA for $1 \mathrm{~h}$ at room temperature. After the BSA solution was aspirated, the samples were incubated with appropriate primary antibodies at $4{ }^{\circ} \mathrm{C}$ overnight. The primary antibodies used in this study were goat anti-dkk1 (sc-14949; Santa Cruz Biotechnology, Santa Cruz, CA, USA), rabbit anti- $\beta$ catenin (RB-1491-P0; NeoMarkers Biotechnology, Fremont, CA, USA), mouse anti-active- $\beta$-catenin (anti-ABC; 05-665; Upstate Biotechnology, Waltham, MA, USA), mouse-anti-BrdU (ZM0013; Zhongshan Biotechnology, Beijing, China) at a concentration of $1 \mu \mathrm{g} / \mathrm{ml}$. After rinsing in PBS thrice, the samples were incubated with FITC-labeled rabbit anti-goat IgG, goat anti-rabbit IgG, and goat anti-mouse IgG respectively (Zhongshan Biotechnology) at $37^{\circ} \mathrm{C}$ for $1 \mathrm{~h}$, then rinsed in PBS. Nuclei were stained with $0.01 \mathrm{mg} / \mathrm{ml}$ propidium iodide (Sigma) or Hoechst 33342 (Sigma) for $10 \mathrm{~min}$. Samples were viewed under a laser scanning confocal microscope (Leica, Heidelberg, Germany). For some samples, preimmune IgG of rabbit, goat, or mouse was used as a negative control.

\section{ELISA}

Decidual cells were isolated and cultured in a six-well plate at a concentration of $1 \times 10^{6}$ cells per well. Culture supernatants from decidual cells were concentrated for 20 times using the Amicon Centricon filter device from Millipore Corporation (Billerica, MA, USA) after culture for $24 \mathrm{~h}$. ELISA was performed using the DuoSet ELISA Development System for mouse Dkk1 (R\&D Systems) according to manufacturer's protocol. The result was measured on a model 3550 Microplate Reader (Bio-Rad Laboratories) at $450 \mathrm{~nm}$. Each experiment contained at least three independent cultures. The values were shown as pg Dkk1/ml supernatant.

\section{EPC proliferation analysis}

To determine the effect of Dkk1 on EPC proliferation, recombinant mouse Dkk1 (200 ng/ml) was added to cultured 
EPCs. After $24 \mathrm{~h}$ of treatment, BrdU (40 $\mu \mathrm{M}$; 10280879001 ; Roche) was added to the medium for $4 \mathrm{~h}$. Cells were then subjected to procedures similar to immunofluorescence staining as mentioned above. Cells migrating from EPCs were quantified by counting the number of BrdU-positive cells in five random fields with Image J software. Each experiment contained at least three independent cultures.

\section{Treatment of pregnant mice with antisense Dkk1 ODN}

Fifty pregnant mice on day 5 were injected with $4 \mu$ l solution containing $20 \mu \mathrm{M}$ antisense Dkk1 ODN into the lumen of one of uterine horn, while the other horn was injected with $4 \mu$ l solution containing $20 \mu \mathrm{M}$ sense ODN as control (sense: $5^{\prime}$-CTT CGG AGA TGA TTG TGT GTG C-3' and antisense: $5^{\prime}$-GCA CAC ACA ACCATCATC TCC GAAG-3'; Li etal. 2007). On day 8, the treated animals were killed and the number of implantation sites was recorded. Ten of these implantation sites having normal configuration were fixed in cold $4 \%$ paraformaldehyde in PBS and embedded in paraffin. Sections cut at $5 \mu \mathrm{m}$ were placed on glass slides coated with poly-L-lysine, deparaffinized, rehydrated, and washed in PBS. Then H\&E staining was performed.

\section{Gelatin zymography}

The EPC culture media were harvested after incubating for $24 \mathrm{~h}$. The media were mixed with $4 \times$ sample buffer $(8 \%$ SDS (w:v), $0.04 \%$ bromophenol blue (w:v), $0.25 \mathrm{M}$ Tris), incubated at $37{ }^{\circ} \mathrm{C}$ for $30 \mathrm{~min}$, and $6 \mu \mathrm{g}$ total protein were subjected to SDS-PAGE in $10 \%$ polyacrylamide gels containing $0.5 \mathrm{mg} / \mathrm{ml}$ gelatin (Difco Laboratories, Detroit, MI, USA). After electrophoresis, the gels were washed twice in 2.5\% Triton X-100 and $50 \mathrm{mM}$ Tris- $\mathrm{HCl}(\mathrm{pH} 7.5)$ at room temperature to remove SDS, and then incubated with calcium assay buffer $(50 \mathrm{mM}$ Tris, $10 \mathrm{mM} \mathrm{CaCl} 2,1 \mu \mathrm{M} \mathrm{ZnCl}_{2}, 1 \%$ Triton X-100, pH 7.5) for $16 \mathrm{~h}$ at $37{ }^{\circ} \mathrm{C}$. The gels were stained with Coomassie Brilliant Blue R-250 in 50\% methanol and $10 \%$ acetic acid for $1 \mathrm{~h}$ at room temperature and destained with $10 \%$ acetic acid. MMPs exhibiting gelatinolytic activity appeared as clear bands. Estimation of molecular weights was possible owing to the concurrent electrophoresis of different molecular weight protein markers. Two wells $(\sim 30$ EPCs per well) were performed per treatment. Each experiment contained at least three independent cultures.

\section{Statistical analysis}

A one-way ANOVA was used for statistical evaluation of the data. Values were presented as mean \pm s.E.M. $P$ values $<0.05$ were considered statistically significant.

\section{Acknowledgements}

We thank Dr Aaron Hsueh (Division of Reproductive Biology, Stanford University) and Tiebang Kang (Department of Cell Biology and Physiology, Washington University in St Louis School of Medicine) for reading the manuscript. This work was supported by the National Basic Research Program of China
(Grant no. 2006CB504006 and 2006CB0F1006) and National Natural Science Foundation of China (Grant no. 30670785). The authors declare that there is no conflict of interest that would prejudice the impartiality of this scientific work.

\section{References}

Abrahamsohn PA \& Zorn TMT 1993 Implantation and decidualization in rodents. Journal of Experimental Zoology 266 603-628.

Afonso S, Romagnano L \& Babiarz B 1997 The expression and function of cystatin C and cathepsin B and cathepsin L during mouse embryo implantation and placentation. Development 124 3415-3425.

Aguilera O, Fraga MF, Ballestar E, Paz MF, Herranz M, Espada J, Garcia JM, Munoz A, Esteller M \& Gonzalez-Sancho JM 2006 Epigenetic inactivation of the Wnt antagonist DICKKOPF-1 (DKK-1) gene in human colorectal cancer. Oncogene 25 4116-4121.

Bischof P, Meisser A \& Campana A 2001 Biochemistry and molecular biology of trophoblast invasion. Annals of the New York Academy of Sciences 943 157-162.

Caluwaerts S, Pijnenborg R, Luyten C \& Van Assche FA 2000 Growth characteristics of diabetic rat ectoplacental cones in vivo and in vitro. Diabetologia 43 939-945.

Cross JC, Werb Z \& Fisher SJ 1994 Implantation and the placenta: key pieces of the developmental puzzle. Science 266 1508-1518.

Dai B, Cao Y, Liu W, Li S, Yang Y, Chen D \& Duan E 2003 Dual roles of progesterone in embryo implantation in mouse. Endocrine 21 123-132.

Enders AC, Chavez DJ \& Schlafke S 1981 Comparison of implantation in utero and in vitro. In Cellular and Molecular Aspects of Implantation, pp 365-382. Eds SR Glassner \& DW Bullock. New York: Plenum Medical.

Faria TN \& Soares MJ 1991 Trophoblast cell differentiation: establishment, characterization, and modulation of a rat trophoblast cell line expressing members of the placental prolactin family. Endocrinology 129 2895-2906.

Foley AC \& Mercola M 2005 Heart induction by Wnt antagonists depends on the homeodomain transcription factor Hex. Genes and Development $19387-396$.

Fritsch MK, Schneider DT, Schuster AE, Murdoch FE \& Perlman EJ 2006 Activation of Wnt/beta-catenin signaling in distinct histologic subtypes of human germ cell tumors. Pediatric and Developmental Pathology 9 115-131.

Gardner RL \& Papaioannou VE 1975 Differentiation in the trophectoderm and inner cell mass. In The Early Development of Mammals, pp 107-132. Ed. M Balls. Cambridge: Cambridge University Press.

Glinka A, Wu W, Delius H, Monaghan AP, Blumenstock C \& Niehrs C 1998 Dickkopf-1 is a member of a new family of secreted proteins and functions in head induction. Nature $391357-362$.

Gumbiner BM 1995 Signal transduction of beta-catenin. Current Opinion in Cell Biology 7 634-640.

Haertel-Wiesmann M, Liang Y, Fantl WJ \& Williams LT 2000 Regulation of cyclooxygenase- 2 and periostin by Wnt-3 in mouse mammary epithelial cells. Journal of Biological Chemistry 275 32046-32051.

Hashimoto H, Itoh M, Yamanaka Y, Yamashita S, Shimizu T, SolnicaKrezel L \& Hibi M 2000 Zebrafish Dkk1 function in forebrain specification and axial mesendoderm formation. Developmental Biology 217 138-152.

Herbst A \& Kolligs FT 2007 Wnt signaling as a therapeutic target for cancer. Methods in Molecular Biology 361 63-91.

Huber O, Korn R, McLaughlin J, Ohsugi M, Herrmann BG \& Kemler R 1996 Nuclear localization of beta-catenin by interaction with transcription factor Lef-1. Mechanism of Development 59 3-10.

Jarvinen E, Salazar-Ciudad I, Birchmeier W, Taketo MM, Jernvall J \& Thesleff I 2006 Continuous tooth generation in mouse is induced by activated epithelial Wnt/beta-catenin signaling. PNAS 103 18627-18632.

Karmakar S, Dhar R \& Das C 2004 Inhibition of cytotrophoblastic (JEG-3) cell invasion by interleukin 12 involves an interferon gamma-mediated pathway. Journal of Biological Chemistry 279 55297-55307.

Khan NI, Bradstock KF \& Bendall LJ 2007 Activation of Wnt/beta-catenin pathway mediates growth and survival in B-cell progenitor acute lymphoblastic leukaemia. British Journal of Haematology 138 338-348. 
Lee AY, He B, You L, Xu Z, Mazieres J, Reguart N, Mikami I, Batra S \& Jablons DM 2004 Dickkopf-1 antagonizes Wnt signaling independent of beta-catenin in human mesothelioma. Biochemical and Biophysical Research Communications 323 1246-1250.

Li J, Liu W, Cao Y, Peng S, Zhang Y \& Duan E 2007 Roles of Dickkopf-1 and its receptor Kremen1 during embryonic implantation in mice. Fertility and Sterility DOI:10.1016/j.fertnstert.2007.09.003.

Mao B, Wu W, Davidson G, Marhold J, Li M, Mechler BM, Delius H, Hoppe D, Stannek P, Walter C et al. 2002 Kremen proteins are Dickkopf receptors that regulate $\mathrm{Wnt} / \beta$-catenin signaling. Nature 417 664-667.

Miller JR \& Moon RT 1996 Signal transduction through beta-catenin and specification of cell fate during embryogenesis. Genes and Development 10 2527-2539.

Mukhopadhyay M, Shtrom S, Rodriguez-Esteban C, Chen L, Tsukui T, Gomer L, Dorward DW, Glinka A, Grinberg A, Huang SP et al. 2001 Dickkopf1 is required for embryonic head induction and limb morphogenesis in the mouse. Developmental Cell 1 423-434.

Peng S, Miao C, Li J, Fan X, Cao Y \& Duan E 2006 Dickkopf-1 induced apoptosis in human placental choriocarcinoma is independent of canonical Wnt signaling. Biochemical and Biophysical Research Communications 350 641-647.

Reya T \& Clevers H 2005 Wnt signalling in stem cells and cancer. Nature 434 843-850.

Romagnano L \& Babiarz B 1990 The role of murine cell surface galactosyltransferase in trophoblast: laminin interactions in vitro. Developmental Biology 141 254-261.
Rothbacher U \& Lemaire P 2002 Creme de la Kremen of Wnt signalling inhibition. Nature Cell Biology 4 E172-E173.

Semenov MV, Tamai K, Brott BK, Kuhl M, Sokol S \& He X $2001 \mathrm{Head}$ inducer Dickkopf-1 is a ligand for Wnt coreceptor LRP6. Current Biology 11 951-961.

Sherman MI \& Atienza-Samols SB 1978 In vitro studies on the surface adhesiveness of mouse blastocysts. In Human Fertilisation, pp 179-183. Eds EH Ludwig \& P Tauber. Boston, MA: PGS.

Vibhakar R, Foltz G, Yoon JG, Field L, Lee H, Ryu GY, Pierson J, Davidson B \& Madan A 2007 Dickkopf-1 is an epigenetically silenced candidate tumor suppressor gene in medulloblastoma. Neuro-Oncology $\mathbf{9}$ 135-144.

van de Wetering M, Sancho E, Verweij C, de Lau W, Oving I, Hurlstone A, van der Horn K, Batlle E, Coudreuse D, Haramis AP et al. 2002 The betacatenin/TCF-4 complex imposes a crypt progenitor phenotype on colorectal cancer cells. Cell 111 241-250.

Wong SC, Chan AT, Chan JK \& Lo YM 2006 Nuclear beta-catenin and Ki-67 expression in choriocarcinoma and its pre-malignant form. Journal of Clinical Pathology 59 387-392.

Received 26 April 2007

First decision 9 May 2007

Revised manuscript received 1 November 2007

Accepted 4 December 2007 